AEI-2010-174, arxiv:1012.4004

overview article: arxiv:1012.3982

\title{
Review of AdS/CFT Integrability, Chapter VI.1: Superconformal Symmetry
}

\author{
NikLAS BeiserT \\ Max-Planck-Institut für Gravitationsphysik, Albert-Einstein-Institut \\ Am Mühlenberg 1, 14476 Potsdam, Germany \\ nbeisert@aei.mpg.de

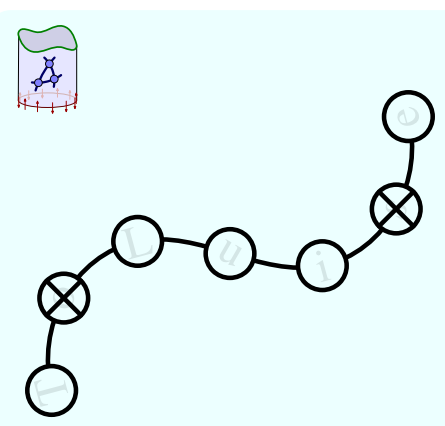

\begin{abstract}
Aspects of the $D=4, \mathcal{N}=4$ superconformal symmetry relevant to the AdS/CFT duality and integrability are reviewed. These include the Lie superalgebra $\mathfrak{p} \mathfrak{s u}(2,2 \mid 4)$, its representations, conformal transformations and correlation functions in $\mathcal{N}=4$ super Yang-Mills theory as well as an illustration of the $A d S_{5} \times S^{5}$ superspace on which the dual string theory is formulated.
\end{abstract}




\section{Introduction}

The AdS/CFT correspondence predicts the exact equivalence of $\mathcal{N}=4$ super YangMills (SYM) theory with IIB superstrings propagating on the $A d S_{5} \times S^{5}$ background. One of the immediate checks is that the two models have coincident global symmetries: $\mathcal{N}=4$ superconformal symmetry on the one hand and the isometries of the $A d S_{5} \times S^{5}$ superspace on the other are both given by the Lie supergroup $\widetilde{\operatorname{PSU}}(2,2 \mid 4)$ or its algebra $\mathfrak{p s u}(2,2 \mid 4)$.

Symmetry serves as an important organising principle - e.g. for objects with similar properties - and leads to structural constraints - e.g. for correlation functions. Furthermore, supersymmetry often implies that selected quantities are protected from receiving quantum corrections. Two famous examples are the exact quantum conformal symmetry of $\mathcal{N}=4 \mathrm{SYM}$ due to absence of a beta-function $[1]$ and the exactness of correlators for certain BPS operators in agreement with a prediction of the AdS/CFT duality,see the review [2]. Nevertheless, agreement of the symmetry groups is far from sufficient to prove an exact duality. ${ }^{1}$ To verify the AdS/CFT conjecture one therefore needs tests involving dynamical quantities which are not protected by the symmetry. Much of the activity concerning AdS/CFT integrability is devoted to such tests. Making use of superconformal symmetry has helped the progress at various stages.

The present paper reviews some aspects of the Lie superalgebra $\mathfrak{p s u}(2,2 \mid 4)$ relevant to AdS/CFT integrability. The presented facts are by no means restricted to integrability; they were known long before AdS/CFT integrability was discovered, and little progress was made in connection with the latter. Nevertheless, many results in AdS/CFT integrability are based on a good knowledge of $\mathfrak{p s u}(2,2 \mid 4)$. This paper therefore serves a different purpose than the other chapters of the review collection $[3]$ : It is not so much a review of one particular aspect of AdS/CFT integrability, but should be viewed as a reference guide to key concepts concerning the underlying global symmetry.

This paper is split into three parts: In Sec. 2 we shall review purely algebraic aspects of $\mathfrak{p s u}(2,2 \mid 4)$ such as the algebra itself as well as some essential representation theory. In Sec. 3 we apply it to local operators in $\mathcal{N}=4 \mathrm{SYM}$ and their correlation functions. In Sec. 4 we discuss the $A d S_{5} \times S^{5}$ background on which superstrings can propagate and which is a particular coset of $\widehat{\operatorname{PSU}}(2,2 \mid 4)$.

\section{The $\mathfrak{p} \mathfrak{s u}(2,2 \mid 4)$ Algebra}

Definition. The algebra $\mathfrak{p s u}(2,2 \mid 4)$ is a real Lie superalgebra of (even|odd) dimension $30 \mid 32$, see e.g. [4]. In order to define it, it is convenient to start with complex 4|4dimensional square supermatrices

$$
X=\left(\begin{array}{l|l}
A & B \\
\hline C & D
\end{array}\right)
$$

\footnotetext{
${ }^{1}$ In this case the large amount of (super)symmetry at least makes both constituent models essentially unique and exceptional, which may be viewed as a hint towards the validity of the correspondence.
} 
Each block $A, B, C, D$ is a $4 \times 4$ matrix of (non-Graßmannian) complex numbers. The blocks $A, D$ are considered even and $B, C$ odd. The Lie superalgebra $\mathfrak{g l}(4 \mid 4, \mathbb{C})$ is the $32 \mid 32$-dimensional vector space of these supermatrices. Its graded Lie bracket $[\cdot, \cdot\}$ is defined as the graded commutator of supermatrices (in the following $Y$ is the analog of $X$ in (2.1) with blocks $E, F, G, H)$

$$
[X, Y\}=X Y-(-1)^{X Y} Y X:=\left(\begin{array}{c|c}
A E+B G-E A+F C & A F+B H-E B-F D \\
\hline C E+D G-G A-H C & C F+D H+G B-H D
\end{array}\right) .
$$

It differs from a conventional commutator through the signs for the odd-odd products $F C$ and $G B$. It also satisfies a graded Jacobi-identity

$$
(-1)^{X Z}[[X, Y\}, Z\}+(-1)^{Y X}[[Y, Z\}, X\}+(-1)^{Z Y}[[Z, X\}, Y\}=0 .
$$

This algebra is not simple, it has non-trivial ideals: One is related to the supertrace $\mathrm{S} \operatorname{Tr} X:=\operatorname{Tr} A-\operatorname{Tr} D$ which is zero for graded commutators $\mathrm{S} \operatorname{Tr}[X, Y\}=0$. Demanding that $\mathrm{S} \operatorname{Tr} X=0$ thus removes a derivation from $\mathfrak{g l}(4 \mid 4, \mathbb{C})$, and restricts it to the subalgebra $\mathfrak{s l}(4 \mid 4, \mathbb{C})$. Furthermore, the identity supermatrix 1 commutes with all other matrices, $[1, X\}=0$. Hence it generates the centre and can be projected out from $\mathfrak{g l}(4 \mid 4, \mathbb{C})$ yielding $\mathfrak{p g l}(4 \mid 4, \mathbb{C})$. The combination of restriction and projection is the 30|32-dimensional complex Lie superalgebra $\mathfrak{p s l}(4 \mid 4, \mathbb{C}) .^{2}$

Real Form. To restrict to the real form $\mathfrak{p s u}(2,2 \mid 4)$ one imposes a hermiticity condition on the supermatrices

$$
\left(\begin{array}{c|c}
A & B \\
\hline C & D
\end{array}\right)=\left(\begin{array}{c|c}
H A^{\dagger} H^{-1} & -i H C^{\dagger} \\
\hline-i B^{\dagger} H^{-1} & D^{\dagger}
\end{array}\right)
$$

where $H$ is a hermitian matrix of signature $(2,2)$. There are two natural choices for $H$ : In the first, $H$ is diagonal, written in terms of $2 \times 2$ blocks $\left('+1 /{ }^{\prime}\right.$ ' denotes the $2 \times 2$ positive/negative identity matrix; $X^{\prime}$ is a reordering of rows and columns to be explained)

$$
H=\left(\begin{array}{cc}
+ & 0 \\
0 & -
\end{array}\right), \quad X=\left(\begin{array}{cc|c}
M_{1} & i N & -i Q_{1} \\
i \bar{N} & M_{2} & +i Q_{2} \\
\hline \bar{Q}_{1} & \bar{Q}_{2} & R
\end{array}\right), \quad X^{\prime}=\left(\begin{array}{c|c|c}
M_{1} & -i Q_{1} & i N \\
\hline \bar{Q}_{1} & R & \bar{Q}_{2} \\
\hline i N & +i Q_{2} & M_{2}
\end{array}\right) .
$$

Here the hermitian blocks $M_{1}$ and $M_{2}$ generate the maximal compact subalgebra $\mathfrak{s u}(2) \oplus$ $\mathfrak{s u}(2) \oplus \mathfrak{u}(1)=\mathfrak{s o}(4) \oplus \mathfrak{s o}(2)$ of $\mathfrak{s u}(2,2)=\mathfrak{s o}(4,2)$. This choice is useful in the context of the $A d S_{5}$ spacetime, cf. Sec. 4, and for unitary representations. Equivalently one can choose an off-diagonal $H$

$$
H=\left(\begin{array}{cc}
0 & + \\
+ & 0
\end{array}\right), \quad X=\left(\begin{array}{cc|c}
L & P & -i Q \\
K & \bar{L} & -i \bar{S} \\
\hline S & \bar{Q} & R
\end{array}\right), \quad X^{\prime}=\left(\begin{array}{c|c|c}
L & -i Q & P \\
\hline S & R & \bar{Q} \\
\hline K & -i \bar{S} & \bar{L}
\end{array}\right)
$$

\footnotetext{
${ }^{2}$ It is not possible to restrict to $\operatorname{Tr} A=\operatorname{Tr} D=0$ because the graded commutator does not close onto such supermatrices; The centre proportional to the unit supermatrix can only be projected out or removed by redefining the graded commutator accordingly.
} 

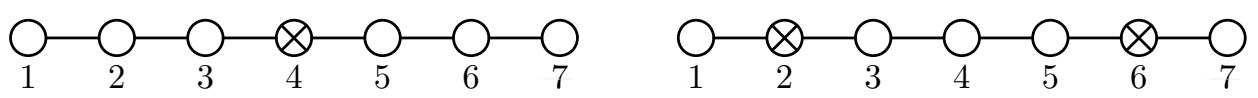

Figure 1: Two Dynkin diagrams for $\mathfrak{s l}(4 \mid 4)=\mathfrak{s l}(2|4| 2)$.

Now the hermitian conjugate blocks $L, \bar{L}$ in $X$ generate the Lorentz and scaling transformations in $\mathfrak{s l}(2, \mathbb{C}) \oplus \mathfrak{g l}(1)=\mathfrak{s o}(3,1) \oplus \mathfrak{s o}(1,1)$. Obviously, this choice is adapted to four-dimensional Minkowski space, see Sec. 3. In the context of the real form $\mathfrak{p s u}(2,2 \mid 4)$ it is often convenient to reorder the 2,2|4 rows and columns, and move one of the 2's past the 4 . The supermatrix $X$ reordered in 2|4|2-block form is displayed in $(2.5 \mid 2.6)$ as $X^{\prime}$. From now on we shall use exclusively the $2|4| 2$-grading.

Simple Generators. A useful presentation of Lie algebras, which is frequently encountered in the solution of integrable systems, is through $r$ triplets of simple (raising, Cartan and lowering) generators $\mathrm{E}_{k}, \mathrm{H}_{k}, \mathrm{~F}_{k},(r$ is the rank of the algebra), see e.g. [5]. For the Lie algebras $\mathfrak{s l}(n)$ the elements $\mathrm{E}_{k}, \mathrm{H}_{k}, \mathrm{~F}_{k}$ with $k=1, \ldots, n-1$, generate the three main diagonals $X_{k, k+1}, X_{k, k}-X_{k+1, k+1}, X_{k+1, k}$. The remaining elements are obtained by repeated Lie brackets, e.g. $\left[\mathrm{E}_{k}, \mathrm{E}_{k+1}\right\}$ generates $X_{k, k+2}$. Evidently, the algebra generated by arbitrary repeated brackets is enormous and needs to be reduced by certain relations. To that end, the simple generators satisfy a set of Chevalley-Serre relations which encode all the information on the specific Lie algebra, $\mathfrak{s l}(n)$, in a condensed form

$$
\begin{gathered}
{\left[\mathrm{H}_{j}, \mathrm{E}_{k}\right\}=+A_{j k} \mathrm{E}_{k}, \quad\left[\mathrm{H}_{j}, \mathrm{~F}_{k}\right\}=-A_{j k} \mathrm{~F}_{k}, \quad\left[\mathrm{E}_{j}, \mathrm{~F}_{k}\right\}=\delta_{j k} \mathrm{H}_{k},} \\
{\left[\left[\mathrm{E}_{k}, \mathrm{E}_{k \pm 1}\right\}, \mathrm{E}_{k \pm 1}\right\}=\left[\left[\mathrm{F}_{k}, \mathrm{~F}_{k \pm 1}\right\}, \mathrm{F}_{k \pm 1}\right\}=0,} \\
{\left[\mathrm{E}_{j}, \mathrm{E}_{k}\right\}=\left[\mathrm{F}_{j}, \mathrm{~F}_{k}\right\}=0 \text { for }|j-k|>1 .}
\end{gathered}
$$

Here $A_{j, k}$ is the Cartan matrix; for $\mathfrak{s l}(n)$ the three main diagonals take the values $-1,+2,-1$ while the other elements are zero. For a superalgebra $\mathfrak{s l}(n \mid m)$ the definition is similar; the main difference is that some of the raising and lowering elements are odd. For an odd $\mathrm{E}_{k}(k=2,6$ in our case $)$ one has to replace the relation $\left[\left[\mathrm{E}_{k \pm 1}, \mathrm{E}_{k}\right\}, \mathrm{E}_{k}\right\}=0$ by two new ones ${ }^{3}$

$$
\left[\mathrm{E}_{k}, \mathrm{E}_{k}\right\}=0, \quad\left[\left[\mathrm{E}_{k-1}, \mathrm{E}_{k}\right\},\left[\mathrm{E}_{k+1}, \mathrm{E}_{k}\right\}\right\}=0,
$$

and similarly for $\mathrm{F}_{k}$. Furthermore for this $k$ two Cartan matrix elements are modified: $A_{k, k}=0$ and $A_{k, k+1}=+1$. Cartan matrices and Chevalley-Serre relations are often displayed in the form of Dynkin diagrams. Two Dynkin diagrams for $\mathfrak{s u}(2,2 \mid 4)$ are displayed in Fig. 1: Dots correspond to simple generators $\mathrm{E}_{k}, \mathrm{H}_{k}, \mathrm{~F}_{k}$; crossed dots indicate odd generators $\mathrm{E}_{k}, \mathrm{~F}_{k}$. Links stand for non-trivial relations between the corresponding simple generators and non-trivial Cartan matrix elements. If two dots $j$ and $k$ are unlinked, the generators $\mathrm{E}_{k}, \mathrm{~F}_{k}, \mathrm{H}_{k}$ and $\mathrm{E}_{j}, \mathrm{~F}_{j}, \mathrm{H}_{j}$ commute and $A_{j k}=0$. Although the two Dynkin diagrams lead to quite different relations, they describe the same algebra.

\footnotetext{
${ }^{3}$ It turns out that in gauge/string applications the latter relations are dropped, see e.g. 6. The new generators $\mathrm{G}_{k} \sim\left[\left[\mathrm{E}_{k-1}, \mathrm{E}_{k}\right\},\left[\mathrm{E}_{k+1}, \mathrm{E}_{k}\right\}\right\}$ (similarly for $\mathrm{F}_{k}$ ) are part of an ideal of a substantially bigger algebra. The ideal generates gauge transformations acting as the constraint $\mathrm{G}_{k} \simeq 0$ for physical states.
} 


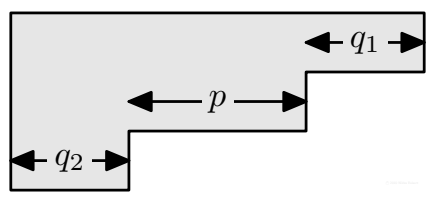

Figure 2: Young diagram corresponding to $\mathfrak{s l}(4)$ representation with Dynkin labels $\left[q_{1}, p, q_{2}\right]$ : A single block corresponds to a fundamental representation, rows and columns correspond to symmetrisation and antisymmetrisation.

The point is that for Lie superalgebras there commonly exist inequivalent choices for the set of simple generators. The two diagrams correspond to the two grading assignments $4 \mid 4$ and $2|4| 2$ for the rows and columns of a supermatrix, cf. $X$ vs. $X^{\prime}$ in $(2.5 \mid 2.6)$.

Unitary Representations. In physical models, multiplets of states transform under unitary representations of the symmetry algebra. Let us therefore review unitary representations of $\mathfrak{p s u}(2,2 \mid 4)|7|$. As the (bosonic part of the) superalgebra is non-compact, unitary representations are necessarily infinite-dimensional. An important class of unitary representations are the lowest-weight (equivalently highest-weight) representations. Under the maximal compact subalgebra $\mathfrak{s u}(2) \oplus \mathfrak{s u}(2) \oplus \mathfrak{s u}(4) \oplus \mathfrak{u}(1)$ such representations decompose into (infinitely many) finite-dimensional irreps, one of which is defined as the lowest. All states corresponding to this lowest irrep are annihilated by the lowering generators associated to the lower triangular blocks $\bar{Q}_{1}, Q_{2}, \bar{N}$ of $X^{\prime}$ in (2.5). The states of the higher irreps arise from the repeated action of the raising generators associated to the upper triangular blocks $Q_{1}, \bar{Q}_{2}, N$ of $X^{\prime}$.

Lowest-weight unitary representations of $\mathfrak{p s u}(2,2 \mid 4)$ are thus specified by an irrep under the maximal compact subalgebra $\mathfrak{s u}(2) \oplus \mathfrak{s u}(2) \oplus \mathfrak{s u}(4) \oplus \mathfrak{u}(1)$. Irreps of the two $\mathfrak{s u}(2)$ 's are specified by their non-negative half-integer spin $\frac{1}{2} s_{1,2}$ or equivalently by the non-negative integer Dynkin labels $\left[s_{1}\right]$ and $\left[s_{2}\right]$. Analogously, irreps of $\mathfrak{s u}(4)$ are specified through three non-negative integer Dynkin labels $\left[q_{1}, p, q_{2}\right]$. An alternative description uses a Young diagram with no more than three rows, see Fig. 2, cf. [8] Finally, a $\mathfrak{u}(1)$ irrep is specified through a number $E$. Here there is a subtlety: The abelian algebra $\mathfrak{u}(1)=\mathbb{R}$ can either generate the compact group $U(1)$ or the non-compact additive group $\mathbb{R}$. For a compact group $E$ is restricted to an integer whereas a non-compact group merely requires $E$ to be real. The supergroup $\operatorname{PSU}(2,2 \mid 4)$ contains the compact version and hence the spectrum of $E$ is discrete. However, $\operatorname{PSU}(2,2 \mid 4)$ has a non-trivial universal cover $\widetilde{\mathrm{PSU}}(2,2 \mid 4)$ where the abelian subgroup becomes non-compact. It is this universal cover which has applications to physics, and consequently we shall allow continuous values for $E$.

Altogether, a unitary representation is specified by the Dynkin labels $\left[s_{1}\right],\left[s_{2}\right]$, $\left[q_{1}, p, q_{2}\right]$ and the number $E$. These combine into $\mathfrak{s u}(2,2 \mid 4)$ Dynkin labels:

$$
\left[s_{1} ; r_{1} ; q_{1}, p, q_{2} ; r_{2} ; s_{2}\right], \quad r_{k}=\frac{1}{2} E+\frac{1}{2} s_{k}-\frac{3}{4} q_{k}-\frac{1}{2} p-\frac{1}{4} q_{3-k} .
$$

Finally, we should note that the value of $E$ must be above a certain bound which is most conveniently expressed in terms of the $r_{k}$

$$
r_{k} \geq 1+s_{k} \quad \text { or } \quad r_{k}=s_{k}=0 .
$$


If one of the bounds for the first condition is saturated or one of the second conditions is satisfied, the representation is called atypical or short. In this case certain combinations of the raising generators annihilate the lowest-weight state. Otherwise there are no additional restrictions on the representation of the raising generators, and the representation is called typical or long.

\section{Superconformal Symmetry in $\mathcal{N}=4 \mathrm{SYM}$}

For $\mathcal{N}=4$ supersymmetric gauge theory on four-dimensional Minkowski space the superPoincaré algebra extends to the superconformal algebra $\mathfrak{p s u}(2,2 \mid 4)$. In the following we shall discuss the representation theory of $\mathfrak{p s u}(2,2 \mid 4)$ related to this gauge theory, see also $[2]$ for an extended review.

Conformal Transformations. Conformal transformations preserve the metric up to a local rescaling of distances. In four-dimensional Minkowski space conformal symmetry is based on the Lie algebra $\mathfrak{s o}(4,2)=\mathfrak{s u}(2,2)$. It contains the $\mathfrak{s l}(2, \mathbb{C})$ Lorentz rotations $\mathrm{L}, \overline{\mathrm{L}}$ and translations $\mathrm{P}$ which form the Poincaré algebra. In addition, there are the dilatation $\mathrm{D}$ and the conformal boosts $\mathrm{K}$. The extension to the superconformal algebra consists of the internal $\mathfrak{s u}(4)$ rotations $\mathrm{R}$, the supertranslations $\mathrm{Q}, \overline{\mathrm{Q}}$ as well as the superconformal boosts $\mathrm{S}, \overline{\mathrm{S}}$. These generators correspond to the submatrices in (2.6).

The conformal generators P, D, K act on the coordinates $x^{\mu}$ of Minkowski space with metric $\eta^{\mu \nu}$ as

$$
\mathrm{P}_{\mu} x^{\nu}=i \delta_{\mu}^{\nu}, \quad \mathrm{D} x^{\mu}=i x^{\mu}, \quad \mathrm{K}^{\mu} x^{\nu}=i x^{\mu} x^{\nu}-\frac{i}{2} \eta^{\mu \nu} x \cdot x .
$$

The action of the odd generators is rather complicated and requires the introduction of fermionic coordinates; we refrain from spelling out the explicit form. Fields on Minkowski space transform according to the above rules, but in addition they have intrinsic transformation properties such as spin and conformal dimension. For example, the conformal representation on a scalar primary field $\Phi(x)$ of dimension $d$ reads

$$
\mathrm{P}_{\mu} \Phi=i \partial_{\mu} \Phi, \quad \mathrm{D} \Phi=i d \Phi+i x \cdot \partial \Phi, \quad \mathrm{K}^{\mu} \Phi=i d x^{\mu} \Phi+i x^{\mu} x \cdot \partial \Phi-\frac{i}{2} x \cdot x \partial^{\mu} \Phi .
$$

Representations for fields with spin are slightly more complicated, and representations of the complete superconformal algebra suggest the use of fields on superspace. Both of these aspects will not be considered explicitly.

Correlators. The power of conformal symmetry is that it constrains correlation functions in a conformal quantum field theory, see e.g. $[9]$. In particular, the spacetime dependence of two- and three-point functions is fully determined

$$
\begin{aligned}
\left\langle\Phi_{1}(x) \Phi_{2}(y)\right\rangle & =\frac{N}{|x-y|^{2 d}}, \quad\left(\text { requires } d_{1}=d_{2}=d\right), \\
\left\langle\Phi_{1}(x) \Phi_{2}(y) \Phi_{3}(z)\right\rangle & =\frac{C_{123}}{|x-y|^{d_{1}+d_{2}-d_{3}}|y-z|^{d_{2}+d_{3}-d_{1}}|z-x|^{d_{3}+d_{1}-d_{2}}} .
\end{aligned}
$$


Indices for fields with spin are typically contracted with suitable tensors, e.g. $I^{\mu \nu}=$ $\eta^{\mu \nu}-2(x-y)^{\mu}(x-y)^{\nu} /(x-y)^{2}$. The reason for complete determination is that any three points can be mapped to any other three points by conformal transformations. The value of the correlator at one configuration of three points thus determines the value of the correlator at any other configuration. For four or more points there exist conformally invariant cross ratios, e.g. $\left|x_{12}\right|\left|x_{34}\right| /\left|x_{13}\right|\left|x_{24}\right|$, on which the correlation functions can depend without constraints. Note that there exist superconformal cross ratios of the fermionic coordinates already for three points in superspace. ${ }^{4}$

The above constraints on correlators hold for all fields which have well-defined transformation properties under superconformal symmetry. This includes the fundamental fields (to some extent), but more importantly also composite local operators. The latter are local products of the fundamental fields and their derivatives. In the free field theory, they transform in tensor products of the fundamental field representation. Let us therefore discuss the superconformal representations that come to use.

Fundamental Field Representation. Consider first a scalar field $\Phi$ in four dimensions. In the free theory $\Phi$ obeys the conformal transformation rules $(3.2)$ with $d=1$. For a local operator we shall need $\Phi$ and its derivatives at the point $x$ which for convenience we assume to be the origin of spacetime $x=0$. In other words, we represent $\Phi(x)$ through its Taylor series around $x=0$

$$
\Phi(x)=\Phi(0)+x^{\mu} \partial_{\mu} \Phi(0)+\frac{1}{2} x^{\mu} x^{\nu} \partial_{\mu} \partial_{\nu} \Phi(0)+\ldots .
$$

We can now see that the conformal representation (3.2) acts on these Taylor components (we drop the argument $x=0$ ):

$$
\begin{aligned}
& \mathrm{P}_{\mu} \Phi=i \partial_{\mu} \Phi, \quad \mathrm{D} \Phi=i d \Phi, \quad \mathrm{K}^{\mu} \Phi=0, \\
& \mathrm{P}_{\mu} \partial_{\rho} \Phi=i \partial_{\rho} \partial_{\mu} \Phi, \quad \mathrm{D} \partial_{\rho} \Phi=i(d+1) \partial_{\rho} \Phi, \quad \mathrm{K}^{\mu} \partial_{\rho} \Phi=i d \delta_{\rho}^{\mu} \Phi,
\end{aligned}
$$

This is a lowest-weight representation, where $\mathrm{K}$ serves as the lowering generator to annihilate the primary field $\Phi$. The raising generator $\mathrm{P}$ is used to access the descendants $\partial_{\mu} \Phi, \partial_{\mu} \partial_{\nu} \Phi, \ldots$, while D essentially measures the number of derivatives.

There is one noteworthy peculiarity of the boost acting on $\partial_{\rho} \partial_{\sigma} \Phi$

$$
\mathrm{K}^{\mu} \partial_{\rho} \partial_{\sigma} \Phi=i(d+1) \delta_{\sigma}^{\mu} \partial_{\rho} \Phi+i(d+1) \delta_{\rho}^{\mu} \partial_{\sigma} \Phi-i \eta_{\rho \sigma} \partial^{\mu} \Phi
$$

When acting on the D'Alembertian derivative $\partial \cdot \partial \Phi$ one obtains $2 i(d-1) \partial^{\mu} \Phi$ which vanishes precisely for the physical scaling dimension $d=1$. This means that the lowestweight representation is reducible, and we should divide out a subrepresentation by imposing the free equation of motion $\partial \cdot \partial \Phi=0$.

The equation of motion implies the absence of certain components in the Taylor expansion. The enumeration of non-trivial components is most transparent when using

\footnotetext{
${ }^{4}$ The number of invariants is related to the dimension of the group, the dimension of the stabiliser and the number of coordinates. E.g., three points in superspace have 48 fermionic coordinates, but the group has only 32 . Hence there should be 16 invariant combinations of fermionic coordinates.
} 
pairs of spinor indices $\beta \dot{\alpha}$ instead of the vector indices $\mu$. Now a trace $\eta^{\mu \nu}$ is replaced by a pair of antisymmetric $\mathfrak{s l}(2, \mathbb{C})$ invariants $\varepsilon^{\beta \delta} \varepsilon^{\dot{\alpha} \dot{\gamma}}$. For any pair of derivatives we can thus exclude antisymmetrisation in both pairs of spinor indices by virtue of the equations of motion. Furthermore, due to the commutative nature of derivatives, antisymmetrisation in just one pair of spinor indices is also zero. Effectively it means that all spinor indices of either kind must be fully symmetrised. Such symmetrisation is automatic for states of a four-dimensional harmonic oscillator: We can replace

$$
\partial_{\beta \dot{\alpha}} \partial_{\delta \dot{\gamma}} \ldots \Phi \simeq \overline{\mathbf{a}}_{\beta} \overline{\mathbf{a}}_{\delta} \ldots \overline{\mathbf{b}}_{\dot{\alpha}} \overline{\mathbf{b}}_{\dot{\gamma}} \ldots|0\rangle
$$

where the algebra of creation and annihilation operators is defined through the non-trivial commutation relations

$$
\left[\mathbf{a}^{\alpha}, \overline{\mathbf{a}}_{\gamma}\right]=i \delta_{\gamma}^{\alpha}, \quad\left[\mathbf{b}^{\dot{\alpha}}, \overline{\mathbf{b}}_{\dot{\gamma}}\right]=i \delta_{\dot{\gamma}}^{\dot{\alpha}}, \quad\left\{\mathbf{c}^{a}, \overline{\mathbf{c}}_{c}\right\}=\delta_{c}^{a} .
$$

Here we have added a set of four fermionic oscillators $\mathbf{c}$ which make the generalisation to all fields of $\mathcal{N}=4$ straight-forward: States have up to four excitations of $\overline{\mathbf{c}}$ transforming in the $\mathfrak{s u}(4)$ representations $\mathbf{1}, \mathbf{4}, \mathbf{6}, \overline{\mathbf{4}}, \mathbf{1}$, respectively. This matches precisely with the representations of the chiral part of the gauge field strength $\Gamma_{\alpha \gamma}$, the chiral fermions $\Psi_{\alpha c}$, the scalars $\Phi_{a c}$, the antichiral fermions $\bar{\Psi}_{\dot{\alpha}}^{c}$ and the antichiral field strength $\bar{\Gamma}_{\dot{\alpha} \dot{\gamma}} \cdot{ }^{5}$ Altogether, for every state of the supersymmetric oscillator, subject to the constraint

$$
\mathbf{N}_{\mathbf{a}}-\mathbf{N}_{\mathbf{b}}+\mathbf{N}_{\mathbf{c}} \simeq 2
$$

there is exactly one Taylor component of the on-shell fundamental fields of $\mathcal{N}=4 \mathrm{SYM}$ [10]. The excitation number operators are defined as $\mathbf{N}_{\mathbf{a}}:=-i \overline{\mathbf{a}}_{\alpha} \mathbf{a}^{\alpha}, \mathbf{N}_{\mathbf{b}}:=-i \overline{\mathbf{b}}_{\dot{\alpha}} \mathbf{b}^{\dot{\alpha}}$, $\mathbf{N}_{\mathbf{c}}:=\overline{\mathbf{c}}_{a} \mathbf{c}^{a}$.

The oscillator basis is also particularly convenient for the superconformal algebra: All the generators are represented through bilinears in the oscillators:

$$
\begin{aligned}
\mathrm{L}^{\alpha}{ }_{\gamma} & \simeq \overline{\mathbf{a}}_{\gamma} \mathbf{a}^{\alpha}-\frac{1}{2} \delta_{\gamma}^{\alpha} \overline{\mathbf{a}}_{\epsilon} \mathbf{a}^{\epsilon}, & \mathrm{R}^{a}{ }_{c} & \simeq \overline{\mathbf{c}}_{c} \mathbf{c}^{a}-\frac{1}{4} \delta_{c}^{a} \overline{\mathbf{c}}_{e} \mathbf{c}^{e}, \\
\overline{\mathrm{L}}_{\dot{\gamma}}{ }^{\dot{\alpha}} & \simeq \mathbf{b}^{\dot{\alpha}} \overline{\mathbf{b}}_{\dot{\gamma}}-\frac{1}{2} \delta_{\dot{\gamma}}^{\dot{\alpha}} \mathbf{b}^{\dot{\epsilon}} \overline{\mathbf{b}}_{\dot{\epsilon}}, & \mathrm{D} & \simeq \frac{1}{2} \overline{\mathbf{a}}_{\alpha} \mathbf{a}^{\alpha}+\frac{1}{2} \mathbf{b}^{\dot{\alpha}} \overline{\mathbf{b}}_{\dot{\alpha}}, \\
\mathrm{P}_{\gamma \dot{\alpha}} & \simeq \overline{\mathbf{a}}_{\gamma} \overline{\mathbf{b}}_{\dot{\alpha}}, & \mathrm{K}^{\gamma \dot{\alpha}} & \simeq \mathbf{b}^{\dot{\alpha}} \mathbf{a}^{\gamma}, \\
\mathrm{Q}_{\gamma}^{a} & \simeq \overline{\mathbf{a}}_{\gamma} \mathbf{c}^{a}, & \mathrm{~S}_{a}^{\gamma} & \simeq \overline{\mathbf{c}}_{a} \mathbf{a}^{\gamma}, \\
\overline{\mathrm{Q}}_{\dot{\gamma} a} & \simeq \overline{\mathbf{c}}_{a} \overline{\mathbf{b}}_{\dot{\gamma}}, & \overline{\mathrm{S}}^{\dot{\gamma} a} & \simeq \mathbf{b}^{\dot{\gamma}} \mathbf{c}^{a} .
\end{aligned}
$$

These satisfy the $\mathfrak{p s u}(2,2 \mid 4)$ algebra along with its reality conditions provided that

$$
\left(\overline{\mathbf{a}}_{\alpha}\right)^{\dagger}=\overline{\mathbf{b}}_{\dot{\alpha}}, \quad\left(\mathbf{a}^{\alpha}\right)^{\dagger}=\mathbf{b}^{\dot{\alpha}}, \quad\left(\overline{\mathbf{c}}_{a}\right)^{\dagger}=\mathbf{c}^{a} .
$$

The algebra extends to $\mathfrak{u}(2,2 \mid 4)$ by introducing a derivation $\mathrm{B} \simeq \overline{\mathbf{c}}_{a} \mathbf{c}^{a}$ and a central charge $\mathrm{C} \simeq-i \overline{\mathbf{a}}_{\alpha} \mathbf{a}^{\alpha}+i \mathbf{b}^{\dot{\alpha}} \overline{\mathbf{b}}_{\dot{\alpha}}+\overline{\mathbf{c}}_{a} \mathbf{c}^{a}$. The constraint (3.9) is equivalent to the vanishing of the central charge, hence the above form a consistent representation of $\mathfrak{p s u}(2,2 \mid 4)$.

${ }^{5}$ The field strength $\Gamma_{\mu \nu}$ with antisymmetric vector indices decomposes into two complex conjugate fields $\Gamma_{\alpha \gamma}$ and $\bar{\Gamma}_{\dot{\alpha} \dot{\gamma}}$ with symmetric spinor indices. Similarly, a real $\mathfrak{s o}(6)$ vector of fields $\Phi_{m}$ is equivalent to a field $\Phi_{a c}$ with antisymmetric $\mathfrak{s u}(4)$ indices and reality condition $\Phi_{a c}=\frac{1}{2} \varepsilon_{a c b d} \bar{\Phi}^{b d}$. 

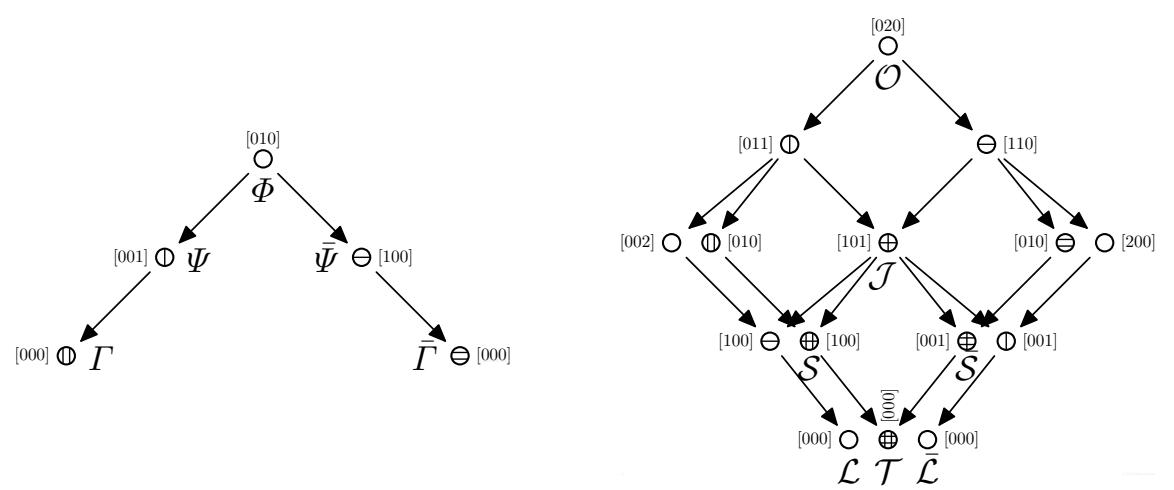

Figure 3: Field multiplet $[0 ; 0 ; 0,1,0 ; 0 ; 0]$ (top component $\Phi$ at $d=1$ ) and current multiplet $[0 ; 0 ; 0,2,0 ; 0 ; 0]$ (top component $\mathcal{O}$ at $d=2$ ). Each dot corresponds to a field of $\mathfrak{s u}(2,2) \oplus \mathfrak{s u}(4)$ : The two $\mathfrak{s u}(2)$ spins are indicated by horizontal/vertical bars, while the $\mathfrak{s u}(4)$ representation is indicated through Dynkin labels. SW/SE arrows correspond to the action of the Poincare supercharges $\mathrm{Q} / \overline{\mathrm{Q}}$.

Note that the above construction remains applicable to the interacting theory for the sake of enumerating local composite operators: The r.h.s. of the equation of motion $\partial \cdot \partial \Phi=\ldots$ is not zero, but it is a product of fields which is already accounted for in the basis of local operators. Furthermore, to maintain proper gauge transformation properties, partial derivatives should be replaced by their covariant counterparts. Consequently, antisymmetries of derivatives are no longer excluded. They lead to commutators with the field strength, which are again accounted for in the basis of local operators. The only change in the quantum theory is that the representation on composite operators is deformed in a specific way, see the chapters [11]. For example, the scaling dimensions of composite operators generically receive continuous quantum corrections.

Composite Operator Multiplets. Composite operators are local products of the fundamental fields and hence they transform in tensor products of the above representation. Tensor products of lowest-weight representations typically decompose into sums of lowest-weight representations. Thus composite operators form multiplets each of which has a primary field.

The simplest non-trivial local operator is a traceless combination of two scalars ${ }^{6}$ $\mathcal{O}_{m n}=\Phi_{m} \Phi_{n}-\frac{1}{6} \delta_{m n} \Phi_{p} \Phi_{p}$ transforming as $(\mathbf{1}, \mathbf{1} ; \mathbf{2 0} ; d=2)$ under $\mathfrak{s l}(2, \mathbb{C}), \mathfrak{s u}(4)$ and dilatations. It is annihilated by $\mathrm{K}, \mathrm{S}, \overline{\mathrm{S}}$ and hence it is the primary field for a multiplet of local operators, cf. Fig. 3. This multiplet is very important because it contains all the conserved currents for $\mathcal{N}=4$ SYM: the $\mathfrak{s u}(4)$ Noether current $\mathcal{J}_{\mu n}^{m}$ transforming as $(\mathbf{2}, \mathbf{2} ; \mathbf{1 5} ; d=3)$, the supersymmetry currents $\mathcal{S}_{\mu \gamma}^{a}, \overline{\mathcal{S}}_{\mu b \dot{\gamma}}$ transforming as $(\mathbf{3}, \mathbf{2} ; \mathbf{4} ; d=3.5)$ and $(\mathbf{2}, \mathbf{3} ; \overline{\mathbf{4}} ; d=3.5)$ and the energy-momentum tensor $\mathcal{T}_{\mu \nu}$ transforming as $(\mathbf{3}, \mathbf{3} ; \mathbf{1} ; d=$ 4). The currents define all Noether charges for $\mathfrak{p s u}(2,2 \mid 4)$, e.g.

$$
R_{b}^{a} \sim \int d^{3} x \mathcal{J}_{0 n}^{m}, \quad Q_{\gamma}^{a} \sim \int d^{3} x \mathcal{S}_{0 \gamma}^{a}, \quad P_{\mu} \sim \int d^{3} x \mathcal{T}_{0 \mu} .
$$

\footnotetext{
${ }^{6}$ In a gauge theory one should pick a gauge-invariant combination.
} 
Moreover, the multiplet contains two scalars $\mathcal{L}_{\text {kin }}, \mathcal{L}_{\text {top }}$ of dimension $d=4$. These are exactly the parity-even kinetic and parity-odd topological parts of the Lagrangian density

$$
\mathcal{L}_{\text {kin }}=-\frac{1}{4} \Gamma^{\mu \nu} \Gamma_{\mu \nu}+\frac{1}{2} \partial^{\mu} \Phi_{m} \partial_{\mu} \Phi_{m}+\ldots, \quad \mathcal{L}_{\text {top }}=\frac{1}{8} \varepsilon^{\mu \nu \rho \sigma} \Gamma_{\mu \nu} \Gamma_{\rho \sigma} .
$$

Next let us consider the labelling of representations for local operators. A lowestweight representation is characterised by its primary field. The latter is characterised by the $\mathfrak{s l}(2, \mathbb{C})$ spin, the $\mathfrak{s u}(4)$ representation and the conformal dimension $d$. For instance the primary field $\Phi_{m}$ of the fundamental field representation transforms as $(\mathbf{1}, \mathbf{1} ; \mathbf{6} ; d=1)$ while the primary field $\mathcal{O}_{m n}$ of the energy-momentum representation transforms as $(\mathbf{1}, \mathbf{1} ; \mathbf{2 0} ; d=2)$. This characterisation is analogous to the discussion of unitary representations of $\mathfrak{s u}(4)$ in Sec. 2 . The only difference is that the representation on the Taylor expansion of local operators is not unitary: ${ }^{7}$ Surely $\mathfrak{s l}(2, \mathbb{C})$ has no finitedimensional unitary representations and also the dilatation generator D has imaginary eigenvalues, cf. (3.5). The point is that the Taylor components are not normalisable in the scalar product defining unitarity. Nevertheless, there is a one-to-one map between representations for local operators and unitary representations. It uses the following complex conformal transformation of Minkowski space

$$
(t, x, y, z) \mapsto 2 r^{-1}(i w, x, y, z) \text { with } w=1-\frac{1}{4} x \cdot x \text { and } r=1-i t+\frac{1}{4} x \cdot x
$$

It maps the dilatation generator to $\mathrm{D} \mapsto i \mathrm{H}$ where $\mathrm{H}$ is the generator of the decompactified $\mathfrak{u}(1)$ discussed in Sec. 2 , so the scaling dimension $d$ maps to the energy eigenvalue $E$. Also the Lorentz algebra $\mathfrak{s l}(2, \mathbb{C})$ is mapped to $\mathfrak{s u}(2) \oplus \mathfrak{s u}(2)$ which is commonly used to classify the spin of fields in four dimensions. For all practical purposes the complex nature of the above conformal transformation is harmless in a perturbative quantum field theory where one commonly continues into complex time directions anyway. Therefore one often works with a dilatation generator $\mathrm{D}^{\prime}=-i \mathrm{D}$ whose spectrum is real and with $\mathfrak{s u}(2) \oplus \mathfrak{s u}(2)$ Lorentz generators $\mathrm{L}^{\prime}$ and $\overline{\mathrm{L}}^{\prime}$. Hence one can classify multiplets of local operators through unitary representation of $\mathfrak{p} \mathfrak{s u}(2,2 \mid 4)$. For instance the fundamental field and energy-momentum multiplets have Dynkin labels $[0 ; 0 ; 0,1,0 ; 0 ; 0]$ and $[0 ; 0 ; 0,2,0 ; 0 ; 0]$, respectively. Note that, the representations $[0 ; 0 ; 0, p, 0 ; 0 ; 0]$ are exceptionally short; the lowest state is annihilated by (at least) half of the supertranslations and hence the multiplet is called half-BPS.

Multiplet Splitting. Scaling dimensions $d$ for unitary representations can take arbitrary real values above a certain unitarity bound, cf. (2.10). Therefore, the scaling dimension typically varies smoothly with the coupling constant of the quantum theory. However, representations at the lower bounds (2.10) have fewer components in general. For example, the scaling dimension for half-BPS representations $[0 ; 0 ; 0, p, 0 ; 0 ; 0]$ is fixed to $d=p$ and cannot depend on the coupling.

Nevertheless, there is an option to combine two or more short representations at the lower bound into a long representation whose scaling dimension can then be increased

${ }^{7}$ Thanks to Gleb Arutyunov and Stefan Fredenhagen for helpful discussions regarding this issue. See also 12 for implications on local operators, correlation functions, string states and their duality. 


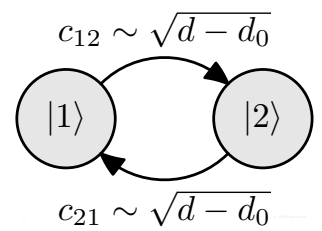

Figure 4: Multiplet splitting at the unitarity bound.

smoothly. This process called multiplet joining (or multiplet splitting in reverse) is an analog of the Higgs effect where a massless vector particle combines with a massless scalar particle to form a massive vector. The set of local operators in $\mathcal{N}=4 \mathrm{SYM}$ has the exceptional feature that almost all short multiplets of the classical theory can be combined into long multiplets in the quantum theory. Only few short multiplets have no partner (such as all half-BPS multiplets $[0 ; 0 ; 0, p, 0 ; 0 ; 0]$ ) and their scaling dimensions are therefore protected from quantum corrections.

Multiplet splitting takes place at the unitarity bound, cf. Fig. 4: Consider a long multiplet which decomposes into two short multiplets at $d=d_{0}$. The representation of some generator $\mathrm{J}$ acts on states $|1\rangle,|2\rangle$ of the submultiplets qualitatively as follows

$$
\mathrm{J}|1\rangle=c_{12}|2\rangle+\ldots, \quad \mathrm{J}|2\rangle=c_{21}|1\rangle+\ldots
$$

The algebra relations imply that $c_{12} c_{21} \sim\left(d-d_{0}\right)$ because splitting at $d=d_{0}$ requires $c_{12}=0$ or $c_{21}=0$. Unitarity furthermore implies $c_{12} \sim c_{21}^{*}$ hence $c_{12} \sim c_{21} \sim \sqrt{d-d_{0}}$. Therefore at $d=d_{0}$ the reality properties of the representation necessarily change, i.e. $d \geq d_{0}$ is a unitarity bound.

\section{Isometries of the $A d S_{5} \times S^{5}$ Superspace}

Supersymmetric strings require a ten-dimensional supergravity background as the space on which they can consistently propagate. Next to a flat spacetime there exist two more maximal supersymmetric backgrounds. One of them is the $A d S_{5} \times S^{5}$ superspace. According to the AdS/CFT correspondence this string theory is exactly dual to conformal $\mathcal{N}=4$ SYM on Minkowski space being the boundary of $A d S_{5} \times S^{5}$, see $\lceil 13$ for an extended review. In the following we shall discuss this superspace, its boundary and its isometries which are generated by the algebra $\mathfrak{p s u}(2,2 \mid 4)$.

$\boldsymbol{A d} \boldsymbol{S}$ Spacetime. We start by defining the anti de Sitter spacetime $A d S_{n+1}$ leaving $n$ generic for the time being. This $(n+1)$-dimensional spacetime has homogeneous negative curvature in close analogy to hyperbolic space $H^{n+1}$. Similar space(time)s with homogeneous positive curvature are the de Sitter spacetime $d S_{n+1}$ and the sphere $S^{n+1}$ (to which we shall frequently contrast $A d S_{n+1}$ ). There are several equivalent constructions which we shall now review. One can embed it into $\mathbb{R}^{n, 2}$ as single-shell hyperboloid specified by

$$
A d S_{n+1}=\left\{X \in \mathbb{R}^{n, 2} \mid X \cdot X=-1\right\}, \quad S^{n+1}=\left\{Y \in \mathbb{R}^{n+2} \mid Y \cdot Y=+1\right\} .
$$


The metric is induced from the flat metric on $\mathbb{R}^{n, 2}$ losing one time-like direction due to the condition $X \cdot X=-1$. An obvious alternative description uses time-like rays $[X]$ in $\mathbb{R}^{n, 2}$

$$
A d S_{n+1}=\left\{[X] \mid X \in \mathbb{R}^{n, 2}, X \cdot X<0\right\}, \quad \text { where }[X]=[Y] \text { iff } X=z Y \text { with } z \in \mathbb{R}^{+} .
$$

The points $X$ or rays $[X]$ transform canonically under $\mathrm{SO}(n, 2)$ and they are stabilised by a $\mathrm{SO}(n, 1)$ subgroup. Consequently, $A d S_{n+1}$ can be viewed as the coset space

$$
A d S_{n+1}=\mathrm{SO}(n, 2) / \mathrm{SO}(n, 1), \quad S^{n+1}=\mathrm{SO}(n+2) / \mathrm{SO}(n+1) .
$$

Thus the group of isometries of $A d S_{5}$ is $\mathrm{SO}(4,2)$. Due to the presence of fermions, one should promote the orthogonal to spin groups. For $n=4$ the group identities $\operatorname{Spin}(4,2)=\operatorname{SU}(2,2)$ and $\operatorname{Spin}(4,1)=\operatorname{Sp}(1,1)$ furthermore allow to write

$$
A d S_{5}=\mathrm{SU}(2,2) / \mathrm{Sp}(1,1), \quad S^{5}=\mathrm{SU}(4) / \mathrm{Sp}(2) .
$$

Coordinates. There exist several choices of coordinates on $A d S_{n+1}$ which are useful in different situations. One is an analog of angle coordinates on the sphere: Using trigonometric functions it is straight-forward to construct a vector $X \in \mathbb{R}^{n, 2}$ with $X \cdot X=$ -1 (we shall use the signature $--+\ldots+$ )

$$
X=(\sec \sigma \cos \tau, \sec \sigma \sin \tau, \tan \sigma \Omega),
$$

where $\Omega \in S^{n-1} \subset \mathbb{R}^{n}$ is a unit vector and $\rho \in\left[0, \frac{1}{2} \pi\right), \tau \in[0,2 \pi)$. The induced metric reads

$$
d s^{2}=\sec ^{2} \sigma\left(d \sigma^{2}-d \tau^{2}\right)+\tan ^{2} \sigma d \Omega^{2} .
$$

On the coordinates $\Omega$ and $\tau$ the maximal compact subgroup $\mathrm{SO}(n) \times \mathrm{SO}(2)$ acts canonically. The remaining $2 n$ directions of $\mathrm{SO}(n, 2)$ act non-trivially.

A useful alternative is Poincaré-type coordinates $x \in \mathbb{R}^{n-1,1}, y \in \mathbb{R}^{+}$with the $\mathbb{R}^{n, 2}$ embedding

$$
X=y^{-1}\left(\frac{1}{2}\left(x \cdot x+y^{2}+1\right), x, \frac{1}{2}\left(x \cdot x+y^{2}-1\right)\right) .
$$

These coordinates reveal the conformally flat nature of the $A d S_{n+1}$ metric

$$
d s^{2}=y^{-2}\left(d x \cdot d x+d y^{2}\right) .
$$

A Poincaré subgroup of $\mathrm{SO}(n, 2)$ acts on the $x$ while the corresponding dilatations act as simultaneous scaling of $x$ and $y$ by the same factor. Special conformal transformations mix up $x$ and $y$ non-trivially

$$
\delta x \sim x(\epsilon \cdot x)-\frac{1}{2} \epsilon\left(x \cdot x+y^{2}\right), \quad \delta y \sim y(\epsilon \cdot x) .
$$

Finally we note that isometries of $A d S_{n+1}$ also include reflections in $\mathbb{R}^{n, 2}$. For example, a reflection in the first component of the above $X$ corresponds to an inversion of time $\tau$ or a conformal inversion of the coordinates $(x, y) \in \mathbb{R}^{n, 1}$

$$
\tau \mapsto \pi-\tau, \quad(x, y) \mapsto-\frac{(x, y)}{x \cdot x+y^{2}} .
$$




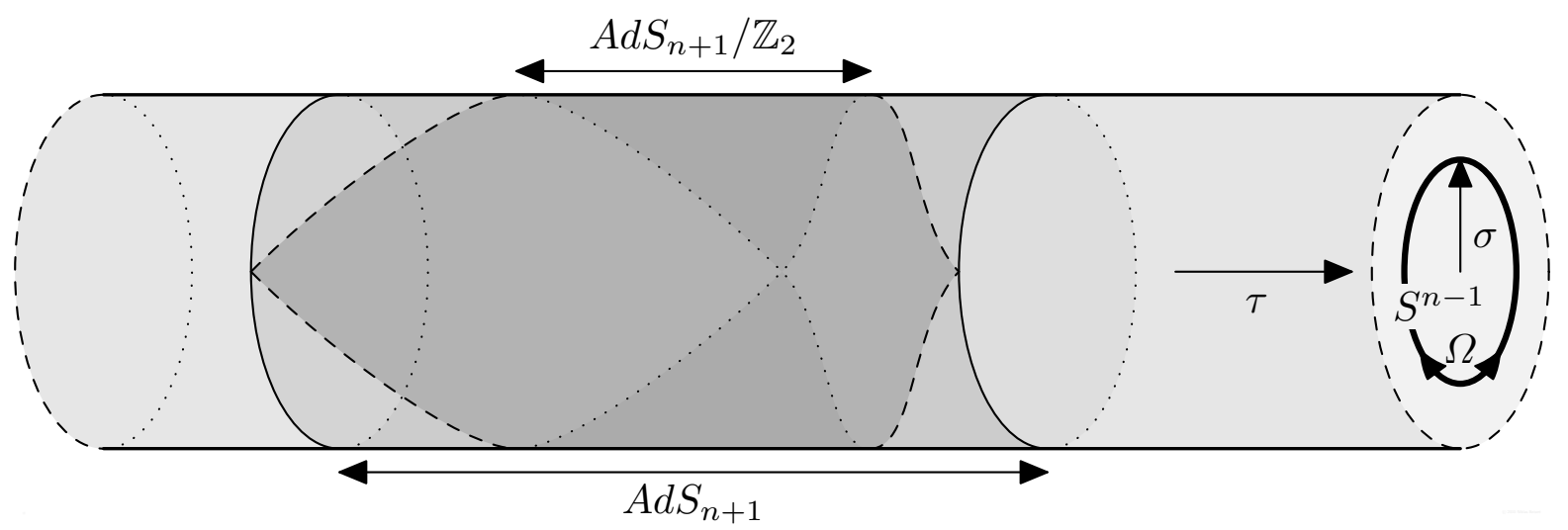

Figure 5: Anti de Sitter space. The infinitely extended solid cylinder represents the universal cover $\overline{A d S_{n+1}}$ (light grey). $A d S_{n+1}$ is obtained by identifying segments of time $\Delta \tau=2 \pi$ (medium grey). The Poincaré patch $A d S_{n+1} / \mathbb{Z}_{2}$ covers half of $A d S_{n+1}$ (dark grey). The boundary $\partial \widetilde{A d S_{n+1}}=\mathbb{R} \times S^{n-1}$ is the outer shell of the cylinder.

Universal Cover. In $(4.5)$ it is clear that the time coordinate $\tau$ is periodic: $\tau \equiv \tau+2 \pi$. Closed time-like curves are inconvenient for physical applications, but luckily they can be removed by lifting to the universal cover $\widetilde{A d S}_{n+1}$ on which a physical model can be defined. Hence the coordinates $(\tau, \sigma, \Omega)$ with non-periodic $\tau \in \mathbb{R}$ define a global chart for $\widetilde{A d S}_{n+1}$ which has the topology of an infinitely extended solid cylinder, see Fig. 5 . The natural embedding into $\mathbb{R}^{n, 2}$ identifies $\tau$ with $\tau+2 \pi \mathbb{Z}$ and leads to $A d S_{n+1}$. Moreover, the Poincaré-type coordinates in (4.7) cover only half of $A d S_{n+1}$. More precisely, if $\theta$ is the angle between $\Omega$ and $\Omega_{0}=(0, \ldots, 0,1)$, then the Poincaré patch is a wedge of the cylinder around $\tau=0$ defined by the inequality $\cos \tau>\sin \sigma \cos \theta$, cf. Fig. 5 .

The universal cover $\widetilde{A d S}_{n+1}$ also has a direct formulation as a coset: The groups $\mathrm{SO}(n, 2)$ and $\mathrm{SU}(2,2)$ have non-trivial coverings because their maximal compact subgroups contain the non-simply connected factors $\mathrm{SO}(2)$ and $\mathrm{U}(1)$, respectively. The covering of $A d S_{n+1}$ is thus defined as

$$
\widetilde{A d S}_{n+1}=\widetilde{\mathrm{SO}}(n, 2) / \operatorname{Spin}(n, 1), \quad \widetilde{\operatorname{Ad} S}_{5}=\widetilde{\mathrm{SU}}(2,2) / \mathrm{Sp}(1,1) .
$$

The universal covers $\widetilde{\mathrm{SO}}(n, 2)$ and $\widetilde{\mathrm{SU}}(2,2)$ are physically relevant because they allow representations with arbitrary real energies as compared to integer values for $\mathrm{SO}(n, 2)$ and $\mathrm{SU}(2,2)$.

Boundary of $\boldsymbol{A d S}$. The boundary $\partial A d S_{n+1}$ of $A d S_{n+1}$ is a $n$-dimensional spacetime. It can be viewed as the space of light-like rays $[X]$ in $\mathbb{R}^{n, 2}$, cf. $(4.2)$

$$
\partial A d S_{n+1}=\left\{[X] \mid X \in \mathbb{R}^{n, 2}, X \cdot X=0\right\}, \quad \text { where }[X]=[Y] \text { iff } X=z Y \text { with } z \in \mathbb{R}^{+} .
$$

In the above coordinates of $A d S_{n+1}$ it is located at $\sigma=\frac{1}{2} \pi$ or at $y=0$. From (4.12) the topology of the $A d S_{n+1}$ boundary follows

$$
\partial A d S_{n+1}=S^{1} \times S^{n-1}, \quad \partial \widetilde{A d S}_{n+1}=\mathbb{R} \times S^{n-1} .
$$


While the topology $S^{1}$ of time in $\partial A d S_{n+1}$ is periodic, the boundary of the universal cover $\widetilde{A d S}_{n+1}$ has no closed time-like curves. Consequently it is the outer shell of the solid cylinder $\widetilde{A d S}_{n+1}$. The metric of $\mathbb{R}^{n, 2}$ can be used to measure angles, but not distances on the boundary, hence it merely induces a conformal metric on $\partial A d S_{n+1}$

$$
d s^{2} \simeq-d \tau^{2}+d \Omega^{2} \simeq d x \cdot d x .
$$

In other words the boundary is conformally flat. This is manifest in the Poincaré coordinates (4.7) $x \in \mathbb{R}^{n-1,1}$ (with $y=0$ ) on which $\widetilde{\mathrm{SO}}(n, 2)$ acts by conformal transformations (3.1).

Note that the boundary is at infinite distance to all points of $A d S_{n+1}$ (similarly to hyperbolic space $H^{n+1}$ and its boundary $\partial H^{n+1}=S^{n}$ ). Nevertheless the boundary can interact with the bulk at finite times: A light ray originating from $\sigma=\tau=0$ reaches the boundary $\sigma=\frac{1}{2} \pi$ at $\tau=\frac{1}{2} \pi$, cf. (4.6). From there it travels back to the point $\sigma=0$ at time $\tau=\pi$.

$\boldsymbol{A d}_{\boldsymbol{5}} \times \boldsymbol{S}^{\mathbf{5}}$ Superspace. The $A d S_{5} \times S^{5}$ superspace is an extension of $\widetilde{A d S_{5}}$ and $S^{5}$ by 32 fermionic directions. It is very conveniently expressed as a coset space: The groups $\mathrm{SU}(2,2)$ and $\mathrm{SU}(4)$ for the definition $A d S_{5}$ and $S^{5}$ in (4.4) combine into the supergroup $\operatorname{PSU}(2,2 \mid 4)$ which has 32 fermionic directions. Dividing by the bosonic denominator groups in (4.4) one obtains the full superspace

$$
\widetilde{A d S}_{5} \times S^{5} \times \mathbb{C}^{0 \mid 16}=\frac{\widetilde{\operatorname{PSU}}(2,2 \mid 4)}{\operatorname{Sp}(1,1) \times \operatorname{Sp}(2)} .
$$

The curvature radii of the $\widetilde{A d S}_{5}$ and $S^{5}$ subspaces are equal but opposite, such that the overall scalar curvature vanishes.

In view of the AdS/CFT correspondence, we shall consider the boundary of this superspace. The sphere $S^{5}$ is closed and the fermionic space $\mathbb{C}^{0 \mid 16}$ has trivial topology such that the overall boundary originates from the $\widehat{A d S}$ factor alone. In the spherical coordinates $(4.5)$, it resides at $\sigma=\frac{1}{2} \pi$. Let us approach the boundary with a codimension-one surface at a fixed $\sigma$ near $\sigma=\frac{1}{2} \pi$. This surface has the topology $\mathbb{R} \times S^{3} \times S^{5} \times \mathbb{C}^{0 \mid 16}$. According to (4.6) the radius of the $S^{3}$ is $\tan \sigma$ while the radius of the $S^{5}$ factor is constantly 1 . Hence at the boundary the $S^{5}$ shrinks to a point in comparison to the $S^{3}$. This means that, for some physical purposes, the boundary of the $A d S_{5} \times S^{5}$ spacetime is effectively the boundary of $\widetilde{A d S}_{5}$ alone, i.e. $\mathbb{R} \times S^{3}$. (A patch of) this spacetime is conformally equivalent to Minkowski space $\mathbb{R}^{3,1}$. The boundary of the $A d S_{5} \times S^{5}$ superspace has additional fermionic coordinates to make up a conformally flat $\mathcal{N}=4$ superspace.

Coset Space Sigma Model. In string theory isometries of the background spacetime become conserved Noether charges. This becomes obvious in the construction of a coset space sigma model, see the chapter [14]. Thus the group of global symmetries of superstrings on $A d S_{5} \times S^{5}$ is $\widetilde{\mathrm{PSU}}(2,2 \mid 4)$. It should be noted that the coset space sigma model construction not only provides the correct target space metric, but also a non-trivial superspace torsion and five-form supergravity flux coupling to the string worldsheet. 
The $A d S_{5} \times S^{5}$ coset has a couple of exceptional features which make it a suitable background for a consistent quantum string theory: First of all, it has 10 bosonic and 32 fermionic coordinates. Furthermore the worldsheet theory on this coset has 16 kappa symmetries to reduce the effective number of fermionic coordinates to 16. Finally, the Killing form for $\operatorname{PSU}(2,2 \mid 4)$ vanishes identically as required for conformal symmetry on the worldsheet. Only few cosets share these features, cf. [15].

\section{References}

[1] M. F. Sohnius and P. C. West, "Conformal Invariance in $\mathcal{N}=4$ Supersymmetric Yang-Mills Theory", Phys. Lett. B100, 245 (1981). S. Mandelstam, "Light Cone Superspace and the Ultraviolet Finiteness of the $\mathcal{N}=4$ Model", Nucl. Phys. B213, 149 (1983). • L. Brink, O. Lindgren and B. E. W. Nilsson, "NN=4 Yang-Mills Theory on the Light Cone", Nucl. Phys. B212, 401 (1983). • P. S. Howe, K. S. Stelle and P. K. Townsend, "Miraculous Ultraviolet Cancellations in Supersymmetry Made Manifest", Nucl. Phys. B236, 125 (1984).

[2] E. D'Hoker and D. Z. Freedman, "Supersymmetric gauge theories and the AdS/CFT correspondence", hep-th/0201253.

[3] N. Beisert et al., "Review of AdS/CFT Integrability: An Overview", arxiv:1012.3982.

[4] J. F. Cornwell, "Group Theory in Physics, Volume III: Supersymmetries and Infinite-Dimensional Algebras", Academic Press (1989), London, UK, Techniques of Physics 10. • L. Frappat, P. Sorba and A. Sciarrino, "Dictionary on Lie Algebras and Superalgebras", Academic Press (2000), London, UK. • L. Frappat, P. Sorba and A. Sciarrino, "Dictionary on Lie Superalgebras", hep-th/9607161.

[5] J. F. Cornwell, "Group Theory in Physics: An Introduction", Academic Press (1997), London, UK.

[6] N. Beisert, “The su(2/3) dynamic spin chain”, Nucl. Phys. B682, 487 (2004), hep-th/0310252.

[7] V. K. Dobrev and V. B. Petkova, "All Positive Energy Unitary Irreducible Representations of Extended Conformal Supersymmetry”, Phys. Lett. B162, 127 (1985).

[8] S. Sternberg, "Group theory and physics", Cambridge University Press (1994), Cambridge, UK.

[9] G. Mack and A. Salam, "Finite component field representations of the conformal group", Ann. Phys. 53, 174 (1969).

[10] M. Günaydin and N. Marcus, "The Spectrum of the $S^{5}$ Compactification of the Chiral $\mathcal{N}$ $=2, D=10$ Supergravity and the Unitary Supermultiplets of U(2,2/4)", Class. Quant. Grav. 2, L11 (1985).

[11] J. A. Minahan, "Review of AdS/CFT Integrability, Chapter I.1: Spin Chains in $\mathcal{N}=4$ SYM", arxiv:1012.3983. • C. Sieg, "Review of AdS/CFT Integrability, Chapter I.2: The spectrum from perturbative gauge theory", arxiv:1012.3984. A. Rej, "Review of AdS/CFT Integrability, Chapter I.3: Long-range spin chains", arxiv:1012.3985.

[12] R. A. Janik, P. Surowka and A. Wereszczynski, "On correlation functions of operators dual to classical spinning string states", JHEP 1005, 030 (2010), arxiv:1002.4613. 
[13] O. Aharony, S. S. Gubser, J. M. Maldacena, H. Ooguri and Y. Oz, "Large $N$ field theories, string theory and gravity", Phys. Rept. 323, 183 (2000), hep-th/9905111.

[14] M. Magro, "Review of AdS/CFT Integrability, Chapter II.3: Sigma Model, Gauge Fixing", arxiv:1012.3988.

[15] K. Zarembo, "Strings on Semisymmetric Superspaces", JHEP 1005, 002 (2010), arxiv: 1003.0465 . 